\title{
The Use of Epidermal Growth Factor Receptor Type 2-Targeting Tyrosine Kinase Inhibitors in the Management of Epidermal Growth Factor Receptor Type 2-Positive Gastric Cancer: A Narrative Review
}

\author{
Asim M. AlMazmomy ${ }^{1}$, Majed M. Al-Hayani ${ }^{2}$, Mohammed Alomari ${ }^{3}$, Abdulrahman G. Bazi ${ }^{4}$ \\ 1. Surgery, College of Medicine King Abdulaziz University, Rabigh, SAU 2. Neurology, College of Medicine King \\ Abdulaziz University, Rabigh, SAU 3. Pediatrics, College of Medicine King Abdulaziz University, Rabigh, SAU 4.
} Internal Medicine, College of Medicine King Abdulaziz University, Rabigh, SAU

Corresponding author: Asim M. AlMazmomy, almazmomyasim@gmail.com

\begin{abstract}
Gastric cancer (GC), including gastroesophageal junction cancer (GEJC), continues to be one of the most frequently diagnosed neoplasms globally. Moreover, GC/GEJC is a principal cause of neoplasm-related fatalities. Early-stage GC/GEJC has a favorable five-year overall survival (OS) rate with surgical resection. However, the vast majority of patients present with advanced inoperable or metastatic disease with a very unfavorable five-year OS rate. Such patients are left with very limited therapeutic options, such as systemic chemotherapy, targeted therapy, and immunotherapy, all of which can be performed as monotherapy or in various combinations. The molecular profiling of GC has revealed several personalized therapeutic vulnerabilities, one of which is the expression of epidermal growth factor receptor type 2 (EGFR2, also known as HER2). HER2 overexpression or amplification is present in a fair subset of patients with GC/GEJC and has been shown to correlate with poor clinicopathological prognostic outcomes. Generally, treatment schemes to tackle HER2 in HER2-positive GC/GEJC comprise the use of anti-HER2 monoclonal antibodies or HER2-targeting tyrosine kinase inhibitors (TKIs). In this study, we engage in a narrative review of the available phase II and III literature on the efficacy and safety of HER2-targeting TKIs in the management of HER2-positive GC/GEJC.
\end{abstract}

Received 11/19/2019

Review began 11/24/2019 Review ended 11/27/2019 Published 12/05/2019

๑) Copyright 2019

AlMazmomy et al. This is an open access article distributed under the terms of the Creative Commons Attribution License CC-BY 3.0., which permits unrestricted use, distribution, and reproduction in any medium, provided the original author and source are credited.
Categories: Gastroenterology, General Surgery, Oncology

Keywords: gastric cancer, gastroesophageal junction cancer, human epidermal growth factor receptor 2, tyrosine kinase inhibitors

\section{Introduction And Background}

Gastric cancer (GC), including gastroesophageal junction cancer (GEJC), is the fifth most commonly diagnosed malignancy and the third leading cause of malignancy-related mortality worldwide [1]. Generally, its incidence is two-fold higher in males than in females [1]. Its incidence frequency is largely concentrated in East Asia, particularly Japan, China, and Korea [1]. The five-year overall survival (OS) rates for GC/GEJC patients with local (stage I-II), regional (stage III), and distant (stage IV) diseases are $68 \%, 31 \%$, and $5 \%$, respectively [2]. Surgical resection is the curative standard of care in patients with early-stage disease. Unfortunately, most patients seek clinical attention when the disease is in an advanced stage in which curative surgical resection is not technically feasible [3]. Patients with primary advanced inoperable, recurrent, or metastatic GC/GEJC usually receive systemic chemotherapy. Even though systemic chemotherapy has shown substantially improved progression-free survival (PFS) and OS rates in patients with GC/GEJC when compared to supportive care, the five-year OS rate still does not extend beyond 20-30\% $[4,5]$. One potential reason for the reduced OS is the inherent heterogeneity of GC/GEJC at the clinical, histological, and molecular levels, which mandates a pressing need to devise personalized treatment approaches in terms of targeted therapy [6].

In line with translating GC/GEJC genomics into targeted therapies, molecular profiling analysis has identified epidermal growth factor receptor type 2 (EGFR2, also known as HER2) as a therapeutic vulnerability [7]. HER2 is one of the four members of the EGFR family of receptor tyrosine kinases. At the cellular level, HER2 is implicated in the regulation of cell proliferation, migration, differentiation, and adhesion. HER2 does not bind to specific ligands, and it is activated through hetero- or homo-dimerization with the other EGFR members, leading to aberrant activation of important oncogenic signaling pathways, such as phosphatidylinositol three-kinase/protein kinase $\mathrm{B} /$ mammalian target of rapamycin (PI3K/AKT/mTOR) [8,9]. HER2 positivity can be established through immunohistochemistry (IHC) and/or fluorescence in situ hybridization (FISH), and it is seen in at least more than $15 \%$ of patients with GC/GEJC $[10,11]$. At the clinical level, HER2 expression is correlated with poor clinicopathological prognostic outcomes, such as old age, advanced-stage disease, large tumor size, high tumor grade, lymphovascular 
space invasion, and poor survival rates [12-15]. Thus, targeting HER2 in HER2-positive GC/GECJ is a plausible therapeutic approach. Of note, HER2 overexpression or amplification in GC/GEJC denotes poor prognosis even in the early stages of GC/GEJC [16]. Therapeutic strategies to target HER2 in HER2-positive GC/GEJC include anti-HER2 monoclonal antibodies and HER2-targeting tyrosine kinase inhibitors (TKIs). The primary aim of this study is to engage in a narrative review of the available phase II and III literature on the efficacy and safety of HER2-targeting TKIs in the management of HER2-positive GC/GEJC.

\section{Review}

\section{Literature search strategy}

We screened the PubMed ${ }^{\circledR}$ database for the period from January 1, 2000 to October 1, 2019 by using the following keywords: "gastric cancer" OR "gastroesophageal junction cancer" OR "HER2 positive" OR "EGFR2 positive” OR "tyrosine kinase inhibitor". Additional references from published articles were also manually screened for potential inclusion in the study analysis. The study inclusion criteria included: (1) studies published in the English language, (2) patients diagnosed with HER2-positive GC/GEJC, (3) studies reporting completed phase II or III trials, and (iv) studies reporting the efficacy and/or safety of the HER2-targeting TKIs lapatinib, afatinib, dacomitinib, and neratinib. For each study included in the review, the following details were also reviewed subject to availability: the first author, year of publication, trial type, trial identification number, study sample size, efficacy, toxicity, duration of follow-up, survival outcomes, and conclusions.

\section{Lapatinib}

Lapatinib is a dual small-molecule TKI which blocks EGFR type 1 (EGFR1, also known as HER1) and HER2 tyrosine kinase activities, resulting in the inhibition of intracellular signaling and suppressing of tumor proliferation [17]. Preclinical studies have demonstrated the in vitro and in vivo efficacy of lapatinib in various HER2-positive GC cells [18]. An earlier study by Iqbal et al. (NCT00103324, Southwest Oncology Group study S0413: a phase II trial) evaluated frontline lapatinib in 33 chemo-naïve patients with advanced or metastatic GC [19]. Patients were divided into two groups according to the median HER2 gene expression level: high $(n=16)$ and low $(n=17)$ median HER2 gene expression. An exploratory analysis showed that patients with high median HER2 gene expression had a statistically significant improved median OS than patients with low HER2 gene expression (6.8 vs. 3.1 months; p: 0.0031).

In 2014, Satoh et al. (NCT00486954: a phase III trial) examined the efficacy and safety of second-line lapatinib plus paclitaxel $(n=132)$ versus paclitaxel alone $(n=129)$ in 261 Asian patients with HER2amplified GC [20]. The lapatinib-plus-paclitaxel group achieved a higher median OS (11 vs. 8.9 months, p: 0.1044), median PFS (5.4 vs. 4.4 months, p: 0.2441), median time-to-progression (TTP: 5.5 vs. 4.4 months, p: 0.2163 ), and overall response rate (ORR: $27 \mathrm{vs.} 9 \%, \mathrm{p}<0.001$ ) than the paclitaxel-alone group. Nevertheless, the clinical improvements in OS, PFS, and TTP achieved by the lapatinib-plus-paclitaxel group were not statistically significant. An exploratory analysis demonstrated that Chinese nationality (as compared to Japanese nationality) and high HER2 IHC positivity (IHC3+ as compared to IHC0/IHC1+/IHC2+) were associated with favorable survival benefits in terms of OS and PFS (all p: <0.05). Treatment-related side effects of any grade were comparable between both groups (100 vs. 98\%, respectively), and most of them were grade-I/-II adverse events. The study concluded that the lapatinib-plus-paclitaxel regimen was not associated with substantially advantageous survival benefits when compared to paclitaxel alone in patients with HER2-amplified GC. Nevertheless, Chinese nationality and HER2 positivity were correlated with better clinical efficacy.

In 2015, Lorenzen et al. (NCT01145404: a phase II trial) investigated the clinical utility and toxicity of second-line lapatinib plus capecitabine $(n=18)$ versus lapatinib alone $(n=19)$ in 37 patients with HER2positive GC [21]. More than half of the patients in both groups had previously received an anti-HER2 monoclonal antibody trastuzumab therapy ( $67 \mathrm{vs.} 63 \%$, respectively). ORR was achieved in two patients (11.1\%) in the lapatinib-plus-capecitabine group, both of which were partial responses. On the other hand, there was no ORR from the lapatinib-alone group (0\%). Median TTP was higher in the lapatinib-pluscapecitabine group than in the lapatinib-alone group ( 83 vs. 42 days). The study was terminated too early due to ineffectiveness. The secondary endpoints of the study of median PFS and OS were largely comparable in both groups (PFS: 47 vs. 41 days; OS: median not reached vs. 142 days). The frequency of treatmentrelated side effects of any grade ( 100 vs. $90 \%$ ) or $\geqslant$ grade-III ( 55.6 vs. $52.6 \%$, respectively) were similar between both groups. However, the incidence of diarrhea was more frequent in the lapatinib-pluscapecitabine group than in the lapatinib-alone group (61 vs. 26\%). The study concluded that second-line lapatinib plus capecitabine was not clinically effective in previously treated patients with HER2-positive GC.

In 2016, Hecht et al. (NCT00680901: a phase III trial) gauged the therapeutic benefits and safety of frontline lapatinib plus capecitabine plus oxaliplatin (lapatinib group, $n=272$ ) versus placebo plus capecitabine plus oxaliplatin (placebo group, $\mathrm{n}=273$ ) in 545 previously untreated patients with advanced or metastatic HER2positive GC/GEJC [22]. Median OS (12.2 vs. 10.5 months, p: 0.3492), median PFS (6 vs. 5.4 months, p: 0.0381 ) and ORR ( 53 vs. $39 \%$, p: 0.0031 ) were higher for the lapatinib group. Nevertheless, the clinical improvement in OS incurred by the lapatinib group was not statistically significant. An exploratory analysis exhibited no statistically significant association between HER2 IHC status and survival. However, Asian patients in the 
lapatinib group had superior median OS than the placebo group (16.5 vs. 10.9 months, p: 0.0261), and this advantageous correlation was demonstrated in a follow-up exploratory analysis as well [23]. Despite modest improvements in OS, PFS, and ORR, the NCT00680901 study concluded that the incorporation of lapatinib to capecitabine plus oxaliplatin did not yield any improved OS benefits in previously untreated patients with advanced or metastatic HER2-positive GC/GEJC.

In 2018, Moehler et al. (NCT01123473: a phase II trial) examined the efficacy of frontline lapatinib plus chemotherapy (lapatinib group, $n=5$ ) versus placebo plus chemotherapy (placebo group, $n=5$ ) in ten patients with metastatic HER2-positive GC [24]. HER2 positivity was confirmed by IHC plus FISH (stratum one) or IHC only (stratum two). Both strata represented the cohort of patients with HER2-positive status in the study. Options for chemotherapy were investigator-chosen and included epirubicin plus cisplatin plus fluorouracil (ECF), or epirubicin plus cisplatin plus capecitabine (ECX). ORR was not substantially different; two patients in the lapatinib group and one patient in the placebo group achieved partial responses. No survival data of PFS and OS were reported. Overall, the study concluded that lapatinib with chemotherapy (ECF or ECX) was not associated with appreciable efficacy in patients with metastatic HER2-positive GC.

Another phase II trial that investigated the efficacy of neoadjuvant lapatinib plus capecitabine plus oxaliplatin in HER2-positive GC patients with liver metastasis has been undertaken (NCT02015169) [25]. However, the results have not yet been made public.

\section{Afatinib}

Afatinib is an irreversible nonspecific small-molecule TKI of the EGFR family. It is approved by the Food and Drug Administration (FDA) as a frontline treatment in patients with activating EGFR mutation-positive nonsmall-cell lung cancer. Afatinib has been shown to exhibit antitumor activities against HER2-positive GC cell lines through the downregulation of HER receptor tyrosine kinases as well as downstream kinase activation [26].

So far, there have been no published phase II or III trials of afatinib in the management of HER2-positive GC/GEJC. Nevertheless, there are two phase II trials currently being conducted to assess the efficacy of afatinib plus paclitaxel as a second-line regimen in trastuzumab-refractory patients with GC/GEJC (NCT01522768 and NCT02501603) [27,28]. Furthermore, there is a completed phase II trial that gauged the efficacy of frontline afatinib plus cisplatin plus fluorouracil in patients with inoperable GC. However, the results are still being analyzed and not yet published (NCT01743365) [29].

\section{Dacomitinib}

Dacomitinib, also recognized as PF-00299804, is a second-generation small-molecule pan-inhibitor of the EGFR family of receptor tyrosine kinases [30]. An earlier preclinical study has reported the anticancer ability of dacomitinib as a monotherapy as well as in combination with chemotherapy/targeted therapy in HER2positive GC cell lines [31].

In 2016, Oh et al. (NCT01152853: a phase II trial) examined the efficacy, toxicity profile, and potential biomarkers of second-line dacomitinib in 27 pretreated patients with HER2-positive GC [32]. Specifically, seven patients (25.9\%) had received prior anti-HER2 treatments. Overall, two (7.4\%) and nine (33.3\%) patients attained partial responses and stable disease, respectively, thus yielding a disease-control rate of 40.7\% (partial responses plus stable disease). The median OS, median PFS, and 4-month PFS rate were 7.1 months, 2.1 months, and $22.2 \%$, respectively. Slightly less than half of the patients $(n=11,40.7 \%)$ exhibited some extent of tumor reduction. The vast majority of drug-related side effects were mild grade-I/-II adverse events, such as fatigue, skin rash, and diarrhea. An exploratory analysis of biomarkers showed that higher serum levels of HER2 extracellular domain and lower levels of soluble E-cadherin as positive prognostic markers of improved efficacy. The study showed that dacomitinib as a second-line agent was associated with acceptable, although not substantial, therapeutic benefits and endurable toxicity profile in previously treated patients with HER2-positive GC. There are no ongoing phase II or III clinical trials about dacomitinib in the management of patients with HER2-positive GC/GEJC.

\section{Varlitinib}

Varlitinib, also recognized as ASLAN001 and ARRY-334543, is a reversible small-molecule TKI of the EGFR family [10].

So far, there are no published phase II or III trials of varlitinib in the management of HER2-positive GC/GEJC. Nevertheless, a phase II trial explored the efficacy of varlitinib in patients with recurrent or metastatic GC. The tumors in these patients were either HER2-amplified or coexpressing HER1 and HER2. However, the results are not yet disseminated (NCT01614522) [33]. On the other hand, there is an ongoing phase II-III trial (NCT03130790) that attempts to explore the therapeutic efficacy and safety of frontline varlitinib plus folinic acid-leucovorin-fluorouracil-oxaliplatin (mFOLFOX6, interventional group) versus placebo plus mFOLFOX6 (placebo group) in patients with chemo-naïve advanced or metastatic HER1- and HER2-positive coexpressing GC [34]. 


\section{Neratinib}

Neratinib, also recognized as HKI-272, is a reversible small-molecule TKI of the EGFR family [10]. A preclinical study showed that neratinib exhibited therapeutic benefits in HER2-positive GC cells [9].

In 2018, Hayman et al. (NCT01953926: a phase II trial) examined the efficacy of neratinib in five patients with HER2-positive GC/GEJC. The results were largely disappointing as no patient achieved ORR and the median PFS achieved was 1.7 months. The study concluded that neratinib did not seem to offer clinical and survival benefits in patients with HER2-positive GC/GEJC. There are no ongoing phase II or III clinical trials about neratinib in the management of patients with HER2-positive GC/GEJC.

\section{Discussion}

The primary goal of molecular profiling and subclassification of GC/GEJC into subtypes is to facilitate the optimal selection of patients in whom molecular targeted therapies can achieve optimum results [6]. HER2 positivity or amplification has been identified in at least a quarter of patients with GC/GEJC [10]. Therapeutic approaches to target HER2 in HER2-positive GC/GEJC include anti-HER2 monoclonal antibodies and HER2-targeting TKIs. The similarities, differences, advantages, and disadvantages of each approach are documented in the literature [36]. HER2-targeting TKIs exhibit their antitumor activities by binding to the adenosine triphosphate (ATP)-binding site of the receptor's intracellular domain, resulting in the inhibition of intracellular signaling and suppressing of tumor proliferation [36]. In this study, we aimed to review the efficacy of HER2-targeting TKIs employed in the management of patients with HER2-positive GC/GEJC.

Table 1 outlines a summary of phase II and III trials that explored HER2-targeting TKIs in the management of patients with HER2-positive GC/GEJC. Collectively, there were six published phase II and III clinical trials: two phase III trials and four phase II trials [20,21,22,24,32,35]. Among them, only two studies included sample sizes of more than 100 patients [20,22]. The setting of HER2-targeting TKI was first-line in two studies, second-line in three studies, and mixed (first-line and second-line) in one study [20,21,32,22,24,35]. Lapatinib was the most commonly reported HER2-targeting TKI $(n=4)$. Lapatinib monotherapy in 19 patients was not associated with favorable survival benefits, in terms of median PFS (1.5 months) and OS (5.1 months) [21]. The combination of lapatinib plus chemotherapy in 422 patients was associated with a mean PFS of 4.4 months (range: 1.7-6 months) and a mean OS of 11.6 months (range: 11-12.2 months. In one study, the median OS was not reached, and the OS was not reported in another one) [20-22,24]. In summary, the overall clinical and survival benefits of HER2-targeting TKIs for lapatinib, dacomitinib, and neratinib were discouraging and not superior to standard-of-care chemotherapy or placebo. 


\section{Cureus}

\begin{tabular}{|c|c|c|c|c|c|c|c|c|c|c|c|c|}
\hline Ref. & $\begin{array}{l}\text { First } \\
\text { author }\end{array}$ & Year & Phase & $\begin{array}{l}\text { Clinical trial } \\
\text { ID }\end{array}$ & Setting & $\begin{array}{l}\text { Sample } \\
\text { size }\end{array}$ & Regimen & $\begin{array}{l}\text { ORR, } \\
\%\end{array}$ & $\begin{array}{l}\text { TTP, } \\
\text { months }\end{array}$ & $\begin{array}{l}\text { PFS, } \\
\text { months }\end{array}$ & $\begin{array}{l}\text { OS, } \\
\text { months }\end{array}$ & $\begin{array}{l}\text { Overall } \\
\text { results }\end{array}$ \\
\hline \multirow{2}{*}{ [20] } & \multirow{2}{*}{ Satoh } & \multirow{2}{*}{2014} & \multirow{2}{*}{ IIII } & \multirow{2}{*}{ NCT00486954 } & \multirow{2}{*}{$\begin{array}{l}\text { Second- } \\
\text { line }\end{array}$} & 132 & Lapatinib + paclitaxel & 27 & 5.5 & 5.4 & 11 & \multirow{2}{*}{ Negative } \\
\hline & & & & & & 129 & Paclitaxel & 9 & 4.4 & 4.4 & 8.9 & \\
\hline \multirow[t]{2}{*}{ [21] } & \multirow[t]{2}{*}{ Lorenzen } & \multirow[t]{2}{*}{2015} & \multirow[t]{2}{*}{ II } & \multirow[t]{2}{*}{ NCT01145404 } & \multirow{2}{*}{$\begin{array}{l}\text { Second- } \\
\text { line }\end{array}$} & 18 & $\begin{array}{l}\text { Lapatinib + } \\
\text { capecitabine }\end{array}$ & 11.1 & 2.9 & 1.7 & $\begin{array}{l}\text { Not } \\
\text { reached }\end{array}$ & \multirow[t]{2}{*}{ Negative } \\
\hline & & & & & & 19 & Lapatinib & 0 & 1.5 & 1.5 & 5.1 & \\
\hline \multirow[t]{2}{*}{ [22] } & \multirow[t]{2}{*}{ Hecht } & \multirow[t]{2}{*}{2016} & \multirow[t]{2}{*}{ IIII } & \multirow[t]{2}{*}{ NCT00680901 } & \multirow{2}{*}{$\begin{array}{l}\text { First- } \\
\text { line }\end{array}$} & 272 & $\begin{array}{l}\text { Lapatinib + } \\
\text { capecitabine + } \\
\text { oxaliplatin }\end{array}$ & 53 & NR & 6 & 12.2 & \multirow[t]{2}{*}{ Negative } \\
\hline & & & & & & 487 & $\begin{array}{l}\text { Placebo + capecitabine } \\
\text { + oxaliplatin }\end{array}$ & 39 & NR & 5.4 & 10.5 & \\
\hline \multirow{2}{*}{ [24] } & \multirow{2}{*}{ Moehler } & \multirow{2}{*}{2018} & \multirow{2}{*}{ II } & \multirow{2}{*}{ NCT01123473 } & \multirow{2}{*}{$\begin{array}{l}\text { First- } \\
\text { line }\end{array}$} & 5 & Lapatinib + ECF/ECX & 40 & NR & NR & NR & \multirow{2}{*}{ Negative } \\
\hline & & & & & & 5 & Placebo + ECF/ECX & 20 & NR & NR & NR & \\
\hline [32] & Oh & 2016 & II & NCT01152853 & $\begin{array}{l}\text { Second- } \\
\text { line }\end{array}$ & 27 & Dacomitinib & 7.4 & NR & 2.1 & 7.1 & Negative \\
\hline [35] & Hayman & 2018 & II & NCT01953926 & Mixed & 5 & Neratinib & 0 & NR & 1.7 & NR & Negative \\
\hline
\end{tabular}

TABLE 1: A summary of the completed phase II and III trials utilizing epidermal growth factor receptor type two (HER2)-targeting tyrosine kinase inhibitors in the management of HER2positive gastric cancer and gastroesophageal cancer

ECF: epirubicin plus cisplatin plus fluorouracil; ECX: epirubicin plus cisplatin plus capecitabine; ID: identifier; NCT: national clinical trial; NR: not reported; ORR: overall response rate; OS: overall survival; PFS: progression-free survival; TTP: time-to-progression

Even though several HER2-targeting TKIs have been developed, their overall therapeutic/prognostic impact on patients has not changed much over time [6]. This can be attributed to the inter- and intra-tumor heterogeneity of HER2-positive GC/GEJC [6]. Moreover, there is an urgent need to identify biomarkers of sensitivity and resistance for these HER2-targeting TKIs in GC/GEJC. Several preclinical studies have identified various biomarkers that confer resistance to lapatinib and afatinib in HER2-positive GC cell lines in vitro and in vivo [37-40,41]. However, these biomarkers have to be validated in human clinical trial studies. In addition, HER2-positive GC patients who were previously treated with anti-HER2 therapy should be inspected for HER2-positive status before the administration of second-line anti-HER2 TKIs. This is because a subset of relapsed patients may undergo conversion from HER2-positive to HER2-negative status [42]. Moreover, it should be noted that the tumor biology of HER2-positive GC/GEJC and HER2-positive breast cancer is not the same, and this may dramatically impact anticancer efficacy. For example, while neratinib offered survival benefits in HER2-positive breast cancer [43], neratinib was largely therapeutically not beneficial in patients with HER2-positive GC/GEJC [35].

Table 2 summarizes the in-progress phase II and III clinical trials of HER2-targeting TKIs in patients with HER2-positive GC/GEJC. 


\section{Cureus}

\begin{tabular}{|c|c|c|c|c|}
\hline Ref & NCT & Phase & Study design & Status \\
\hline [25] & NCT02015169 & II & $\begin{array}{l}\text { Neoadjuvant lapatinib plus capecitabine plus oxaliplatin in HER2-positive GC patients with liver } \\
\text { metastasis }\end{array}$ & $\begin{array}{l}\text { Completed, } \\
\text { not published }\end{array}$ \\
\hline [27] & NCT01522768 & II & $\begin{array}{l}\text { Afatinib plus paclitaxel as second-line therapy in patients with advanced HER2-positive } \\
\text { trastuzumab-refractory advanced GEJC }\end{array}$ & Ongoing \\
\hline [28] & NCT02501603 & II & Afatinib plus paclitaxel as second-line therapy in patients with advanced HER2-positive GC & Ongoing \\
\hline [29] & NCT01743365 & II & Frontline afatinib plus cisplatin plus 5-fluorouracil in patients with inoperable GC & $\begin{array}{l}\text { Completed, } \\
\text { not published }\end{array}$ \\
\hline [33] & NCT01614522 & II & $\begin{array}{l}\text { Varlitinib in patients with recurrent/metastatic GC whose tumors are either HER2-amplified or } \\
\text { coexpressing HER1 and HER2 }\end{array}$ & $\begin{array}{l}\text { Completed, } \\
\text { not published }\end{array}$ \\
\hline [34] & NCT03130790 & II-III & $\begin{array}{l}\text { Frontline varlitinib plus mFOLFOX6 vs. placebo plus mFOLFOX6 in chemo-naîve patients with } \\
\text { advanced/metastatic HER1- and HER2-positive coexpressing GC }\end{array}$ & Ongoing \\
\hline
\end{tabular}

TABLE 2: A summary of completed (but not published) and ongoing phase II/III trials that use epidermal growth factor receptor type two (HER2)-targeting tyrosine kinase inhibitors in the management of HER2-positive gastric cancer and gastroesophageal cancer

GC: gastric cancer; GEJC: gastroesophageal junction cancer; HER1: epidermal growth factor receptor type one; HER2: epidermal growth factor receptor type two; mFOLFOX6: modified folinic acid plus 5-fluorouracil plus oxaliplatin; NCT: national clinical trial; Ref: reference

Our study has two primary limitations that should be acknowledged. First, limiting our literature search to the PubMed ${ }^{\circledR}$ database only (as opposed to other databases) and opting for a narrative review (as opposed to a systematic review) research design might have resulted in a failure to include all potential published phase II and III trials. Second, the very few numbers of the published phase II and III trials $(\mathrm{n}=5)$, the heterogeneity of study settings, and the small sample sizes of patients made it difficult to conduct a metaanalysis and deduce concrete conclusions about the utility of HER2-targeting TKIs in the management of patients with HER2-positive GC/GEJC.

\section{Conclusions}

In patients with HER2-positive GC/GEJC, molecular targeted therapy using current HER2-targeting TKIs (lapatinib, afatinib, dacomitinib, and neratinib) largely does not yield substantial clinical and survival benefits. Thus, the utility of current HER2-targeting TKIs in the management of HER2-positive GC/GEJC is questionable. As it stands, anti-HER2 monoclonal antibodies are preferred over HER2-targeting TKIs in the management of HER2-positive GC/GEJC. Prospective research may include synthesis of new HER2-targeting TKIs or identification of synergistic combinations utilizing old HER2-targeting TKIs plus/minus cytotoxic chemotherapy plus/minus additional molecular targeted therapy plus/minus immunotherapy. The ultimate goal should be to develop HER2-targeting regimens that are capable of offering maximum therapeutic efficacy with minimum side effects.

\section{Additional Information \\ Disclosures}

Conflicts of interest: In compliance with the ICMJE uniform disclosure form, all authors declare the following: Payment/services info: All authors have declared that no financial support was received from any organization for the submitted work. Financial relationships: All authors have declared that they have no financial relationships at present or within the previous three years with any organizations that might have an interest in the submitted work. Other relationships: All authors have declared that there are no other relationships or activities that could appear to have influenced the submitted work.

\section{References}

1. Bray F, Ferlay J, Soerjomataram I, Siegel RL, Torre LA, Jemal A: Global cancer statistics 2018: GLOBOCAN estimates of incidence and mortality worldwide for 36 cancers in 185 countries. CA Cancer J Clin. 2018, 68:394-424. 10.3322/caac.21492

2. Cancer stat facts: stomach cancer. (2019). Accessed: November 15, 2019: https://seer.cancer.gov/statfacts/html/stomach.html.

3. Verdecchia A, Corazziari I, Gatta G, Lisi D, Faivre J, Forman D; EUROCARE Working Group: Explaining gastric cancer survival differences among European countries. Int J Cancer. 2004, 109:737-741. 10.1002/ijc. 20047 
4. Wagner AD, Unverzagt S, Grothe W, Kleber G, Grothey A, Haerting J, Fleig WE: Chemotherapy for advanced gastric cancer. Cochrane Database Syst Rev. 2010, CD004064. Accessed: December 4, 2019 : 10.1002/14651858.CD004064.pub3

5. Yazici O, Sendur MA, Ozdemir N, Aksoy S: Targeted therapies in gastric cancer and future perspectives . World J Gastroenterol. 2016, 22:471-489. 10.3748/wjg.v22.i2.471

6. Bonelli P, Borrelli A, Tuccillo FM, Silvestro L, Palaia R, Buonaguro FM: Precision medicine in gastric cancer. World J Gastrointest Oncol. 2019, 11:804-829. Accessed: December 4, 2019: 10.4251/wjgo.v11.i10.804

7. Ang YL, Yong WP, Tan P: Translating gastric cancer genomics into targeted therapies . Crit Rev Oncol Hematol. 2016, 100:141-146. 10.1016/j.critrevonc.2016.02.007

8. Coussens L, Yang-Feng TL, Liao YC, et al.: Tyrosine kinase receptor with extensive homology to EGF receptor shares chromosomal location with neu oncogene. Science. 1985, 230:1132-1139. 10.1126/science.2999974

9. Hamzehlou S, Momeny M, Zandi Z, et al.: Anti-tumor activity of neratinib, a pan-HER inhibitor, in gastric adenocarcinoma cells. Eur J Pharmacol. 2019, 863:172705. 10.1016/j.ejphar.2019.172705

10. Selim JH, Shaheen S, Sheu WC, Hsueh CT: Targeted and novel therapy in advanced gastric cancer . Exp Hematol Oncol. 2019, 8:25. Accessed: December 4, 2019: 10.1186/s40164-019-0149-6

11. Kim MA, Lee HS, Lee HE, Jeon YK, Yang HK, Kim WH: EGFR in gastric carcinomas: prognostic significance of protein overexpression and high gene copy number. Histopathology. 2008, 52:738-746. 10.1111/j.13652559.2008.03021.x

12. Park DI, Yun JW, Park JH, et al.: HER-2/neu amplification is an independent prognostic factor in gastric cancer. Dig Dis Sci. 2006, 51:1371-1379. 10.1007/s10620-005-9057-1

13. Rakhshani N, Kalantari E, Bakhti H, Sohrabi MR, Mehrazma M: Evaluation of HER-2/neu overexpression in gastric carcinoma using a tissue microarray. Asian Pac J Cancer Prev. 2014, 15:7597-7602. 10.7314/apjcp.2014.15.18.7597

14. Begnami MD, Fukuda E, Fregnani JH, Nonogaki S, Montagnini AL, da Costa WL Jr, Soares FA: Prognostic implications of altered human epidermal growth factor receptors (HERs) in gastric carcinomas: HER2 and HER3 are predictors of poor outcome. J Clin Oncol. 2011, 29:3030-3036. 10.1200/JCO.2010.33.6313

15. Gamboa-Dominguez A, Dominguez-Fonseca C, Quintanilla-Martinez L, et al.: Epidermal growth factor receptor expression correlates with poor survival in gastric adenocarcinoma from Mexican patients: a multivariate analysis using a standardized immunohistochemical detection system. Mod Pathol. 2004, 17:579-587. 10.1038/modpathol.3800085

16. Curea FG, Hebbar M, Ilie SM, Bacinschi XE, Trifanescu OG, Botnariuc I, Anghel RM: Current targeted therapies in HER2-positive gastric adenocarcinoma. Cancer Biother Radiopharm. 2017, 32:351-363. 10.1089/cbr.2017.2249

17. Voigtlaender M, Schneider-Merck T, Trepel M: Lapatinib. Recent Results Cancer Res. 2018, 211:19-44. 10.1007/978-3-319-91442-8_2

18. Wainberg ZA, Anghel A, Desai AJ, et al.: Lapatinib, a dual EGFR and HER2 kinase inhibitor, selectively inhibits HER2-amplified human gastric cancer cells and is synergistic with trastuzumab in vitro and in vivo. Clin Cancer Res. 2010, 16:1509-1519. 10.1158/1078-0432.ccr-09-1112

19. Iqbal S, Goldman B, Fenoglio-Preiser CM, et al.: Southwest Oncology Group study S0413: a phase II trial of lapatinib (GW572016) as first-line therapy in patients with advanced or metastatic gastric cancer. Ann Oncol. 2011, 22:2610-2615. 10.1093/annonc/mdr021

20. Satoh T, Xu RH, Chung HC, et al.: Lapatinib plus paclitaxel versus paclitaxel alone in the second-line treatment of HER2-amplified advanced gastric cancer in Asian populations: TyTAN--a randomized, phase III study. J Clin Oncol. 2014, 32:2039-2049. 10.1200/JCO.2013.53.6136

21. Lorenzen S, Riera Knorrenschild J, Haag GM, et al.: Lapatinib versus lapatinib plus capecitabine as secondline treatment in human epidermal growth factor receptor 2-amplified metastatic gastro-oesophageal cancer: a randomised phase II trial of the Arbeitsgemeinschaft Internistische Onkologie. Eur J Cancer. 2015, 51:569-576. 10.1016/j.ejca.2015.01.059

22. Hecht JR, Bang YJ, Qin SK, et al.: Lapatinib in combination with capecitabine plus oxaliplatin in human epidermal growth factor receptor 2-positive advanced or metastatic gastric, esophageal, or gastroesophageal adenocarcinoma: TRIO-013/LOGiC--a randomized phase iii trial. J Clin Oncol. 2016, 34:443-451. 10.1200/JCO.2015.62.6598

23. Press MF, Ellis CE, Gagnon RC, et al.: HER2 status in advanced or metastatic gastric, esophageal, or gastroesophageal adenocarcinoma for entry to the TRIO-013/LOGiC trial of lapatinib. Mol Cancer Ther. 2017, 16:228-238. 10.1158/1535-7163.MCT-15-0887

24. Moehler M, Schad A, Maderer A, et al.: Lapatinib with ECF/X in the first-line treatment of metastatic gastric cancer according to HER2neu and EGFR status: a randomized placebo-controlled phase II study (EORTC 40071). Cancer Chemother Pharmacol. 2018, 82:733-739. 10.1007/s00280-018-3667-8

25. Phase II study of neoadjuvant XELOX + lapatinib in HER2(+) gastric cancer patients with liver metastasis . (2018). Accessed: November 23, 2019: https://clinicaltrials.gov/ct2/show/NCT02015169.

26. Keller S, Zwingenberger G, Ebert $\mathrm{K}$, et al.: Effects of trastuzumab and afatinib on kinase activity in gastric cancer cell lines. Mol Oncol. 2018, 12:441-462. 10.1002/1878-0261.12170

27. Afatinib and paclitaxel in patients with advanced HER2-positive trastuzumab-refractory advanced esophagogastric cancer. (2019). Accessed: November 23, 2019: https://clinicaltrials.gov/ct2/show/NCT01522768.

28. Afatinib, paclitaxel, 2nd line, advanced gastric cancer . (2019). Accessed: November 23, 2019: https://clinicaltrials.gov/ct2/show/NCT02501603.

29. Clinical trial of chemotherapy combination cisplatin-fluorouracil-afatinib in patients with inoperable gastric cancer (A-GAPP). (2019). Accessed: November 23, 2019: https://clinicaltrials.gov/ct2/show/NCT01743365.

30. Malapelle U, Ricciuti B, Baglivo S, et al.: Osimertinib. Recent Results Cancer Res. 2018, 211:257-276. 10.1007/978-3-319-91442-8_18

31. Nam HJ, Ching KA, Kan J, et al.: Evaluation of the antitumor effects and mechanisms of PF00299804, a pan- 
HER inhibitor, alone or in combination with chemotherapy or targeted agents in gastric cancer. Mol Cancer Ther. 2012, 11:439-451. 10.1158/1535-7163.MCT-11-0494

32. Oh DY, Lee KW, Cho JY, Kang WK, Im SA, Kim JW, Bang YJ: Phase II trial of dacomitinib in patients with HER2-positive gastric cancer. Gastric Cancer. 2016, 19:1095-1103. 10.1007/s10120-015-0567-z

33. A clinical trial evaluating the effect of ASLAN001 in patients with recurrent/metastatic gastric cancer whose tumors are either HER-2 amplified or co-expressing HER-1 and HER-2. (2015). Accessed: November 23, 2019: https://clinicaltrials.gov/ct2/show/NCT01614522.

34. Varlitinib in combination with mFOLFOX6 for advanced or metastatic gastric cancer (first line) . (2019). Accessed: November 23, 2019: https://clinicaltrials.gov/ct2/show/NCT03130790.

35. Hyman DM, Piha-Paul SA, Won H, et al.: HER kinase inhibition in patients with HER2- and HER3-mutant cancers. Nature. 2018, 554:189-194. 10.1038/nature25475

36. Dassonville O, Bozec A, Fischel JL, Milano G: EGFR targeting therapies: monoclonal antibodies versus tyrosine kinase inhibitors. Similarities and differences. Crit Rev Oncol Hematol. 2007, 62:53-61. 10.1016/j.critrevonc.2006.12.008

37. Kim HP, Han SW, Song SH, et al.: Testican-1-mediated epithelial-mesenchymal transition signaling confers acquired resistance to lapatinib in HER2-positive gastric cancer. Oncogene. 2014, 33:3334-3341. 10.1038/onc.2013.285

38. Xiang Z, Song S, Zhu Z, et al.: LncRNAs GIHCG and SPINT1-AS1 are crucial factors for pan-cancer cells sensitivity to lapatinib. Front Genet. 2019, 10:25. Accessed: December 4, 2019: 10.3389/fgene.2019.00025

39. Yu Y, Yu X, Liu H, Song Q, Yang Y: miR494 inhibits cancer-initiating cell phenotypes and reverses resistance to lapatinib by downregulating FGFR2 in HER2-positive gastric cancer. Int J Mol Med. 2018, 42:998-1007. 10.3892/ijmm.2018.3680

40. Park J, Choi Y, Ko YS, et al.: FOXO1 suppression is a determinant of acquired lapatinib-resistance in HER2positive gastric cancer cells through MET upregulation. Cancer Res Treat. 2018, 50:239-254. 10.4143/crt.2016.580

41. Yoshioka T, Shien K, Takeda T, et al.: Acquired resistance mechanisms to afatinib in HER2-amplified gastric cancer cells. Cancer Sci. 2019, 110:2549-2557. 10.1111/cas.14089

42. Seo S, Ryu MH, Park YS, et al.: Loss of HER2 positivity after anti-HER2 chemotherapy in HER2-positive gastric cancer patients: results of the GASTric cancer HER2 reassessment study 3 (GASTHER3). Gastric Cancer. 2019, 22:527-535. 10.1007/s10120-018-0891-1

43. Chan A, Delaloge S, Holmes FA, et al.: Neratinib after trastuzumab-based adjuvant therapy in patients with HER2-positive breast cancer (ExteNET): a multicentre, randomised, double-blind, placebo-controlled, phase 3 trial. Lancet Oncol. 2016, 17:367-377. 10.1016/S1470-2045(15)00551-3 